\title{
Random Time-Scale Invariant Diffusion and Transport Coefficients
}

\author{
Y. He, ${ }^{1}$ S. Burov, ${ }^{1}$ R. Metzler, ${ }^{2}$ and E. Barkai ${ }^{1}$ \\ ${ }^{1}$ Department of Physics, Bar Ilan University, Ramat-Gan 52900, Israel \\ ${ }^{2}$ Physics Department, Technical University of Munich, D-85747 Garching, Germany
}

(Received 29 April 2008; published 28 July 2008)

\begin{abstract}
Single particle tracking of mRNA molecules and lipid granules in living cells shows that the time averaged mean squared displacement $\overline{\delta^{2}}$ of individual particles remains a random variable while indicating that the particle motion is subdiffusive. We investigate this type of ergodicity breaking within the continuous time random walk model and show that $\overline{\delta^{2}}$ differs from the corresponding ensemble average. In particular we derive the distribution for the fluctuations of the random variable $\overline{\delta^{2}}$. Similarly we quantify the response to a constant external field, revealing a generalization of the Einstein relation. Consequences for the interpretation of single molecule tracking data are discussed.
\end{abstract}

DOI: 10.1103/PhysRevLett.101.058101

PACS numbers: 87.10.Mn, 02.50. $-\mathrm{r}, 05.40 . \mathrm{Fb}$

An ensemble of noninteracting Brownian particles spreads according to Fick's law as a Gaussian packet. The ensemble averaged mean square displacement (MSD) is $\left\langle x^{2}(t)\right\rangle=2 D_{1} t$ where $D_{1}$ is the diffusion constant. By an Einstein relation $D_{1}$ is expressed in terms of statistical properties of the microscopic jumps according to $D_{1}=\left\langle\delta x^{2}\right\rangle / 2\langle\tau\rangle$ where $\langle\tau\rangle$ is the average time between jumps and $\left\langle\delta x^{2}\right\rangle$ is the variance of the jump lengths. Instead, one can analyze the time series $x(t)$ of the particle trajectory and determine the time averaged (TA) MSD

$$
\overline{\delta^{2}}(\Delta, t)=\frac{\int_{0}^{t-\Delta}\left[x\left(t^{\prime}+\Delta\right)-x\left(t^{\prime}\right)\right]^{2} d t^{\prime}}{t-\Delta},
$$

where $\Delta$ is called the lag time. For regular Brownian motion and long measurement time $t \gg\langle\tau\rangle$ we have $\overline{\delta^{2}}=$ $2 D_{1} \Delta$, i.e., an ergodic behavior such that the diffusion coefficient obtained from an individual trajectory is identical to the diffusion constant found from an ensemble of particles under identical physical conditions.

From in vivo single particle tracking the diffusion of lipid granules in yeast cells [1] and of mRNA molecules in E. coli cells [2] two findings were made. (i) The TA MSD is subdiffusive, $\overline{\delta^{2}} \sim 2 \bar{D}_{\alpha} \Delta^{\alpha}$ with $\alpha \approx 3 / 4$. Usually subdiffusion is defined by the behavior of an ensemble of particles $\left\langle x^{2}(t)\right\rangle=2 D_{\alpha} t^{\alpha} / \Gamma(1+\alpha)$ and $0<\alpha<1$. Such anomalous behavior is widespread [3-7], including charge carrier transport in amorphous semiconductors [4], models of gene regulation [8], enzymatic binding in crowded cellular environments [9], and anomalous dynamics of cell migration [10] to name but a few. (ii) The second striking observation [1,2] was that the TA diffusion coefficient $\bar{D}_{\alpha}$ is a random variable different from the diffusion constant of the ensemble $D_{\alpha}$, albeit the measurement time is long (see below) [11]. Namely using Eq. (1) to compute a TA MSD we get a result which varies from one single particle trajectory to another [1,2] (see Fig. 4). This means that ergodicity is broken such that time and ensemble averages of the diffusion process are nonidentical.
In this Letter we investigate a widely applicable model for anomalous diffusion: the continuous time random walk (CTRW) [3-7]. In CTRW subdiffusion is scale invariant and $\langle\tau\rangle \rightarrow \infty$ which naturally leads to ergodicity breaking $[12,13]$. We show that for the subdiffusive CTRW the TA MSD (1) differs from the ensemble average, even in the limit of long averaging times. We obtain the distribution of TA MSDs that completely quantifies the magnitude of the new fluctuations. Then we treat the biased random walk showing that the TA response to an external driving field $F$ also remains random. These new findings lead to a new type of fluctuation-dissipation relation for anomalous kinetics which depends both on the lag time $\Delta$ and the measurement time $t$. Finally, we discuss the validity of our theory in experimental situations and its generality in other models of anomalous diffusion.

The uncoupled CTRW in one dimension is considered [3-6]. The probability density function (PDF) of jump lengths is $f(\delta x)$ for which we assume that its variance $\left\langle\delta x^{2}\right\rangle=\int_{-\infty}^{\infty} \delta x^{2} f(\delta x) d(\delta x)$ is finite. Waiting times between jump events are distributed with a common PDF $\psi(\tau)$. So the particle waits in its initial location for a random waiting time, then makes a jump in space, and then the process is renewed. Our main interest is in the case where the average sojourn time is infinite $\langle\tau\rangle=\infty$, namely the subdiffusive case with a power law $\operatorname{PDF} \psi(\tau) \sim$ $A \tau^{-(1+\alpha)} /|\Gamma(-\alpha)|$ and $0<\alpha<1$. Physical models which give specific values of $\alpha$ for different systems and models are given in Refs. [3-6].

We simulate CTRW trajectories with $\alpha=3 / 4$, for an unbiased random walk on a lattice $f(x)=[\delta(x-1)+$ $\delta(x+1)] / 2$ and in Fig. 1 show the TA MSD (1) of 10 individual trajectories with free boundary conditions. The most striking feature in the figure is that the curves are nonidentical, and the TA MSDs remain a random variable even though a large number of jump events occur. In contrast, if we choose a waiting time distribution with $\alpha>$ 1 the TAs will be identical to the ensemble average and nonrandom when the measurement time is long. 
To develop a theory for the observed behavior we first consider the average of Eq. (1) for unbiased CTRWs, namely for the case when the average jump length is zero. We consider first free boundary conditions, a widely applicable case [3-7], not necessarily relevant for the bounded motion in the cell (see below). We have $x\left(t^{\prime}+\right.$ $\Delta)-x\left(t^{\prime}\right)=\sum_{i=1}^{\left.n_{(t,} t^{\prime}+\Delta\right)} \delta x_{i}$, where $\left\{\delta x_{i}\right\}$ are random jump lengths, and $n_{\left(t^{\prime}, t^{\prime}+\Delta\right)}$ is the number of jumps in the interval $\left(t^{\prime}, t^{\prime}+\Delta\right)$. For the unbiased CTRW the $\left\{\delta x_{i}\right\}$ 's are independent random variables with zero mean; hence, $\left\langle\left[x\left(t^{\prime}+\right.\right.\right.$ $\left.\left.\Delta)-x\left(t^{\prime}\right)\right]^{2}\right\rangle=\left\langle\delta x^{2}\right\rangle\left\langle n_{\left(t^{\prime}, t^{\prime}+\Delta\right)}\right\rangle$. The average number of jumps in $\left(t^{\prime}, t^{\prime}+\Delta\right)$ is $\left\langle n_{\left(t^{\prime}, t^{\prime}+\Delta\right)}\right\rangle=\left\langle n_{\left(0, t^{\prime}+\Delta\right)}\right\rangle-\left\langle n_{\left(0, t^{\prime}\right)}\right\rangle$. Using $\left\langle n_{\left(0, t^{\prime}\right)}\right\rangle \sim t^{\prime \alpha} /[A \Gamma(1+\alpha)]$,

$$
\left\langle\overline{\delta^{2}}\right\rangle=\frac{\left\langle\delta x^{2}\right\rangle}{A \Gamma(1+\alpha)} \frac{t^{1+\alpha}-\Delta^{1+\alpha}-(t-\Delta)^{1+\alpha}}{(1+\alpha)(t-\Delta)}
$$

is obtained from Eq. (1). In the limit $\Delta \ll t$ we find

$$
\left\langle\overline{\delta^{2}}\right\rangle \sim \frac{2 D_{\alpha}}{\Gamma(1+\alpha)} \frac{\Delta}{t^{1-\alpha}},
$$

where we used the generalized Einstein relation $D_{\alpha}=$ $\left\langle\delta x^{2}\right\rangle /(2 A)$ [14]. For $\alpha \neq 1$ Eq. (3) is very different from the behavior found for an ensemble, $\left\langle x^{2}(t)\right\rangle=$ $2 D_{\alpha} t^{\alpha} / \Gamma(1+\alpha)$ indicating ergodicity breaking. Equation (3) shows that if we know through measurement the ensemble averaged anomalous diffusion coefficient $D_{\alpha}$ we can determine the single particle trajectory averaged behavior. The result Eq. (3) can be explained by noting that the longer the process goes on, the more likely we are to find long trapping times of the order of the measurement time (aging). Hence, $\left\langle\overline{\delta^{2}}\right\rangle$ decreases when measurement time $t$ is increased. Roughly speaking the diffusion constant depends on time $D(t) \sim d\left\langle x^{2}\right\rangle / d t \sim t^{\alpha-1}$ and Eq. (3) is described by $\overline{\delta^{2}} \simeq D(t) \Delta$ so a linear dependence on the lag time also seen in the simulations in Fig. 1 is found.

Distribution of $\overline{\delta^{2}}$. - For the CTRW under investigation we still have the usual scaling of $x^{2} \sim N$ with the number of jumps $N$ in $(0, t)$, however, due to the broad distribution of waiting times $N \sim t^{\alpha}$ and so $x^{2} \sim t^{\alpha}$. Similar scaling arguments can be used to analyze the distribution of $\overline{\delta^{2}}$. Assume no jump event occurs between time $t_{1}$ and $t_{2}$ and that $t_{2} \gg t_{1}+\Delta$. Then for $t_{1}<t^{\prime}<t_{2}-\Delta$ we have $\left[x\left(t^{\prime}+\Delta\right)-x\left(t^{\prime}\right)\right]^{2}=0$. Since for the scale free dynamics we have long sojourn times of the order of the measurement time without any jump event, $\left[x\left(t^{\prime}+\Delta\right)-x\left(t^{\prime}\right)\right]^{2}=$ 0 for long renewal periods separated by shorter periods of activity. The most important point to realize is that for the process $\left[x\left(t^{\prime}+\Delta\right)-x\left(t^{\prime}\right)\right]^{2}$ the distribution of sojourn times in state $\left[x\left(t^{\prime}+\Delta\right)-x\left(t^{\prime}\right)\right]^{2}=0$ follows the same power law decay as the original process $x(t)$ with a waiting time $\operatorname{PDF} \psi(\tau) \sim \tau^{-(1+\alpha)}$. This means that when $N$ serves as the operational time we have normal behavior

$$
\overline{\delta^{2}} \sim C N / t,
$$

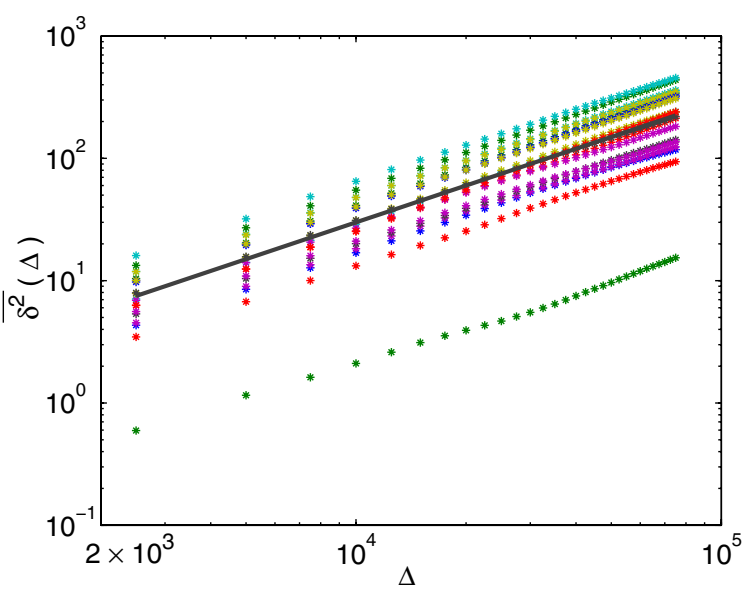

FIG. 1 (color online). Simulations of the subdiffusive CTRW process with $\alpha=3 / 4$ and free boundary conditions show that the TA MSD is a random variable depending on individual trajectories. The solid curve is the averaged behavior Eq. (3). The measurement time is $t=10^{8}$ and $\psi(\tau)=\alpha \tau^{-(1+\alpha)}$ for $\tau>1$.

where $C$ is a constant independent of $N$ soon to be determined, and in the denominator we approximate $t-\Delta \sim t$. Let $P_{N}(t)$ be the probability of making $N$ jumps in the time interval $(0, t)$ and $\hat{P}_{N}(u)$ its Laplace transform. From the convolution theorem $\hat{P}_{N}(u)=[1-\hat{\psi}(u)] \times$ $\exp [N \ln \hat{\psi}(u)] / u$ as well known [14]. Since we are interested in the long time behavior only the small $u$ expansion $\hat{\psi}(u) \sim 1-A u^{\alpha}$ is relevant and we have

$$
\hat{P}_{N}(u) \sim A u^{\alpha-1} \exp \left(-N A u^{\alpha}\right) .
$$

Inverting to the time domain

$$
P_{N}(t) \sim \frac{t}{\alpha A^{1 / \alpha} N^{1+1 / \alpha}} l_{\alpha}\left(\frac{t}{A^{1 / \alpha} N^{1 / \alpha}}\right),
$$

where $l_{\alpha}(t)$ is the one-sided Lévy stable PDF, whose Laplace pair is $\exp \left(-u^{\alpha}\right)[15,16]$. To find $C$ we note that after averaging $\left\langle\overline{\delta^{2}}\right\rangle=C\langle N\rangle / t$, using $\langle N\rangle \sim t^{\alpha} / A \Gamma(1+\alpha)$ and Eq. (3) we have $C=2 A D_{\alpha} \Delta$. By change of variables we obtain the PDF of the dimensionless random variable $\xi=\overline{\delta^{2}} /\left\langle\overline{\delta^{2}}\right\rangle$ using Eqs. (4) and (6)

$$
\lim _{t \rightarrow \infty} \phi_{\alpha}(\xi)=\frac{\Gamma^{1 / \alpha}(1+\alpha)}{\alpha \xi^{1+1 / \alpha}} l_{\alpha}\left[\frac{\Gamma^{1 / \alpha}(1+\alpha)}{\xi^{1 / \alpha}}\right] .
$$

This is one of our main results since it describes the distribution of a large class of time average observables, as we soon show. When $\alpha \rightarrow 1$ we have an ergodic behavior $\lim _{\alpha \rightarrow 1} \phi_{\alpha}(\xi)=\delta(\xi-1)$. A measure of ergodicity breaking (EB) is the parameter

$$
\mathrm{EB}=\lim _{t \rightarrow \infty} \frac{\left\langle\left(\overline{\delta^{2}}\right)^{2}\right\rangle-\left\langle\overline{\delta^{2}}\right\rangle^{2}}{\left\langle\overline{\delta^{2}}\right\rangle^{2}}=\frac{2 \Gamma^{2}(1+\alpha)}{\Gamma(1+2 \alpha)}-1,
$$

which is independent of the lag time $\Delta$ and $D_{\alpha}$. 
In Fig. 2 we show the behavior of the average of $\overline{\delta^{2}}$ and the fluctuations characterized by the EB parameter, showing excellent agreement between asymptotic theory and simulations though the convergence of the EB parameter is typically slow. In Fig. 3 simulations of the PDF of $\overline{\delta^{2}} /\left\langle\overline{\delta^{2}}\right\rangle$ for $\alpha=1 / 2$ and $\alpha=3 / 4$ are shown. We see that for $\alpha=$ $3 / 4$ we have a peak close to $\overline{\delta^{2}} /\left\langle\overline{\delta^{2}}\right\rangle=1$ which indicates that we are closer to the ergodic phase $(\alpha \rightarrow 1)$ while for $\alpha=1 / 2$ the peak is on zero indicating stronger nonergodic behavior as we decrease $\alpha$.

Biased CTRW and generalized Einstein relation.-Now we assume that $\langle\delta x\rangle \neq 0$ but constant, a case which leads to anomalous drift. We consider the TA

$$
\bar{\delta}(\Delta, t)=\int_{0}^{t-\Delta}\left[x\left(t^{\prime}+\Delta\right)-x\left(t^{\prime}\right)\right] d t^{\prime} /(t-\Delta) .
$$

First we obtain the average which is done with an approach similar to the unbiased case, and using $\left\langle x\left(t^{\prime}+\Delta\right)-\right.$ $\left.x\left(t^{\prime}\right)\right\rangle=\langle\delta x\rangle\left[\left(t^{\prime}+\Delta\right)^{\alpha}-t^{\prime \alpha}\right] /[A \Gamma(1+\alpha)]$ we find

$$
\langle\bar{\delta}\rangle \sim \frac{\langle\delta x\rangle}{A \Gamma(1+\alpha)} \frac{\Delta}{t^{1-\alpha}},
$$

for $t \gg \Delta$. Then we can show that the PDF of $\xi=\bar{\delta} /\langle\bar{\delta}\rangle$ is given by Eq. (7); thus, fluctuations of the TA MSD of the unbiased random walk and the fluctuations of the biased mean response have identical distributions.

As is well known according to the generalized Einstein relation the transport of an ensemble of particles is related to the free diffusion of the same particles by $\langle x(t)\rangle_{F}=$ $F\left\langle x^{2}(t)\right\rangle / 2 k_{b} T$, where $\left\langle x^{2}(t)\right\rangle$ is the ensemble average MSD in the absence of a force field and $\langle x(t)\rangle_{F}$ is the mean drift when a constant force $F$ is applied to the system [3,17-19]. This relation can be used to prove that on a microscopic scale $\langle\delta x\rangle_{F}=F\left\langle\delta^{2} x\right\rangle /\left(2 k_{b} T\right)$, which can be obtained from
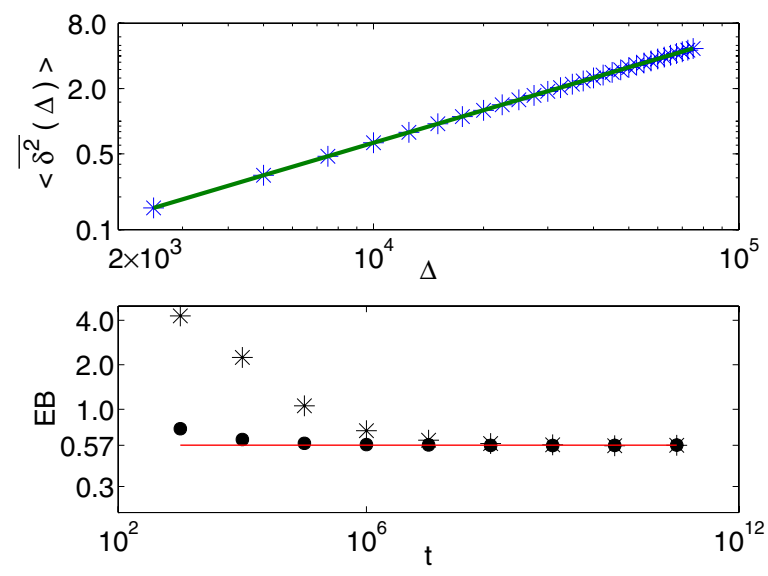

FIG. 2 (color online). (a) $\left\langle\overline{\delta^{2}}\right\rangle$ versus $\Delta$ for $\alpha=1 / 2$ and $t=$ $10^{8}$. Stars are simulations and the solid curve is theory Eq. (3) without fitting. (b) The EB parameter converges slowly to the asymptotic value $\mathrm{EB}=0.5708$ given by Eq. (8). Here $\Delta=10$ (dots), $\Delta=2500$ (stars), and $\alpha=1 / 2$. thermal detailed balance conditions [17]. Using this relation and Eqs. (3) and (10)

$$
\langle\bar{\delta}\rangle_{F}=F\left\langle\overline{\delta^{2}}\right\rangle /\left(2 k_{b} T\right)
$$

which is valid under usual linear response assumptions. This Einstein relation for the TAs while clearly related to the Einstein relation for the ensemble average is valid for any lag time $\Delta$ and measurement time $t$. In this sense it differs from the usual Einstein relation. As mentioned, the relation between transport and diffusion runs deeper, at least within the CTRW model, since we showed that the fluctuations are identical as long as the external field does not modify $\psi(\tau)$ (a reasonable assumption for weak fields [17]) and described by Eq. (7).

Relation with experiments. - Our results can be tested in single particle experiments, for example, for a bead anomalously diffusing in an actin network which exhibits a CTRW type of dynamics [20]. However, in the experiments in the cell $[1,2]$ the particle motion is bounded by the cell walls. Indeed the particles may interact with the cell wall many times whenever $2 D_{\alpha} t^{\alpha} / \Gamma(1+\alpha)>L^{2}$, where $L$ is the system length. Finiteness of the system implies that at long times the ensemble averaged MSD will not increase with time but rather saturate.

We have simulated the effect of a boundary by considering an unbiased CTRW on a lattice with system size $L=$ 62 with lattice spacing equal unity and $\alpha=3 / 4$. The simulations shown in Fig. 4 look similar to experiment and we have $\overline{\delta^{2}} \simeq \Delta^{\beta}$ with $\beta \simeq 3 / 4$ at least within a reasonable time window. The exponent $\beta<1$ depends on the system size, on $\alpha$, and on the time window under investigation. Still our numerical results show that CTRW theory is compatible with available experiment. A direct test of our theory would be to change the experimental time

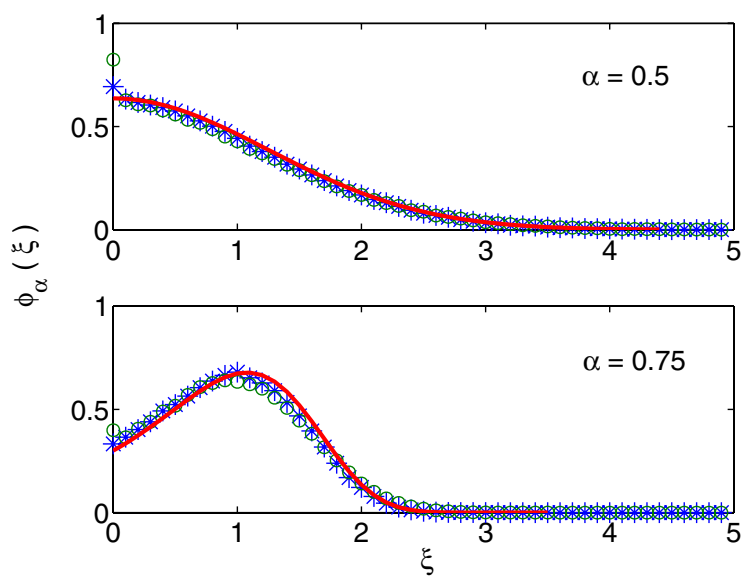

FIG. 3 (color online). PDF of the scaled random variable $\xi=$ $\overline{\delta^{2}} /\left\langle\overline{\delta^{2}}\right\rangle$ for $\alpha=1 / 2$ and $\alpha=3 / 4$ with $t=10^{8}$ and $t=10^{7}$, respectively. The full line is Eq. (7). Stars $(\Delta=2500)$ and circles $\left(\Delta=4 \times 10^{4}\right)$ are simulations. 


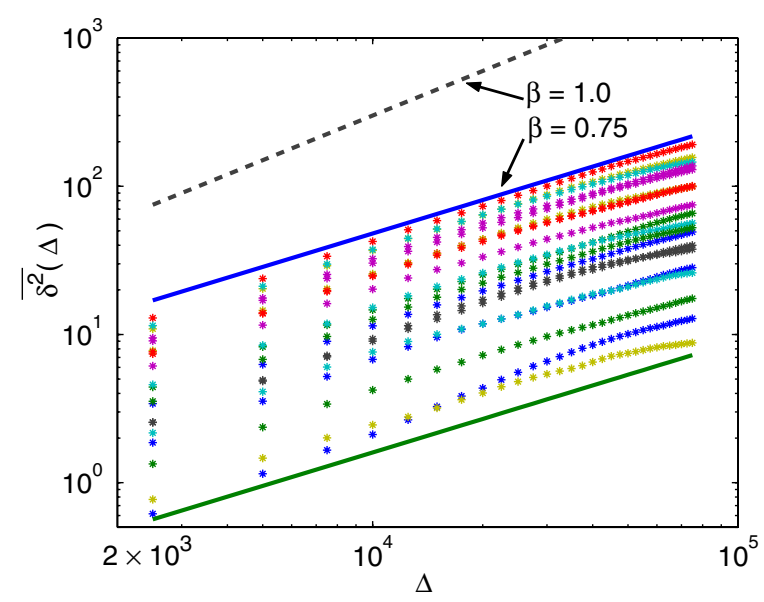

FIG. 4 (color online). Time average $\overline{\delta^{2}}$ vs $\Delta$ for unbiased CTRW on a lattice of size $L=62$, mimicking a particle bounded in a finite domain, as found in the cell. Compared with the unbounded case in Fig. 1 the diffusion is slower. The trajectory averaged MSD follows $\overline{\delta^{2}} \sim \Delta^{\beta}$ with $\beta=3 / 4$ similar to what is found in [1,2]. The measurement time was $t=10^{8}, \alpha=3 / 4$.

$t$ (not only $\Delta$ as done so far) and see if the TA diffusion slows down with increasing $t$.

Notice that for free boundary conditions we get $\overline{\delta^{2}} \propto \Delta$, the existence of the boundaries thus causes the diffusion to appear slower (i.e., $\beta<1$ ) which is intuitively expected. For free boundary conditions, what appears as normal diffusion in a single particle measurement therefore may actually be a hidden subdiffusive process. For both free and reflecting boundary conditions as we increase the measurement time diffusion is slowed down when the TA procedure is made. In all such experiments it is thus imperative to analyze the TA MSD also as function of the measurement time $t$. Additional clues about the nature of the diffusion are the potential scatter of the diffusivity as well as the shape of the trajectories.

In the cell, the measurement time $t$ is limited by the lifetime of the cell. This is important from the point of view of theory which usually assumes an infinite measurement time. Indeed in a finite volume one can expect from a thermodynamical argument demanding stationarity that if $\psi(\tau)$ decays like a power law it does so only within a finite time interval and then a cutoff will appear. However, the finite lifetime of the cell implies that the usual long time limit essential for ergodicity may not be reached and ergodicity breaking is found: Ergodicity of diffusion processes is not fulfilled in a living cell.

More generally we expect TA diffusion and transport coefficients to remain random in other models of anomalous diffusion. We have recently shown [21] that for intermittent weakly chaotic systems exhibiting anomalous diffusion, the distribution of scaled TA Lyaponov exponents is described by Eq. (7); similar behavior is found for superdiffusive Lévy walks. For random walks in random environments (e.g., random trap and comb models) we expect similar behavior due to the deep connections between these models and CTRW theory (in these models $\langle\tau\rangle \rightarrow \infty$ as in the subdiffusive CTRW; however, the disorder is quenched not annealed). Thus one of the most basic paradigms of transport and diffusion theory, namely that information obtained from single particle tracking is contained already in the ensemble measurement, is not valid for anomalous diffusion. This has ramifications for vast classes of processes.

This work was supported by the Israel Science Foundation. We thank Ido Golding and Lene Oddershede for discussions.

[1] I. M. Tolić-Nørrelykke et al., Phys. Rev. Lett. 93, 078102 (2004).

[2] I. Golding and E. C. Cox, Phys. Rev. Lett. 96, 098102 (2006).

[3] J.P. Bouchaud and A. Georges, Phys. Rep. 195, 127 (1990).

[4] H. Scher, M. F. Shlesinger, and J. T. Bendler, Phys. Today 44, No. 1, 26 (1991).

[5] R. Metzler and J. Klafter, Phys. Rep. 339, 1 (2000).

[6] Anomalous Transport: Foundations and Applications, edited by R. Klages, G. Radons, and I. M. Sokolov (Wiley-VCH, Weinheim, 2007).

[7] S. Condamin et al., Proc. Natl. Acad. Sci. U.S.A. 105, 5675 (2008).

[8] M. A. Lomholt, I. M. Zaid, and R. Metzler, Phys. Rev. Lett. 98, 200603 (2007).

[9] S. B. Yuste and K. Lindenberg, Phys. Rev. E 76, 051114 (2007).

[10] P. Dietrich, R. Klages, R. Preuss, and A. Schwab, Proc. Natl. Acad. Sci. U.S.A. 105, 459 (2008).

[11] Scatter of local diffusivities in single particle tracking was also observed by M. Platani, I. Goldberg, A. I. Lamond, and J. R. Swedlow, Nat. Cell Biol. 4, 502 (2002).

[12] J. P. Bouchaud, J. Phys. I 2, 1705 (1992).

[13] G. Bel and E. Barkai, Phys. Rev. Lett. 94, 240602 (2005); A. Rebenshtok and E. Barkai, ibid. 99, 210601 (2007) show that at equilibrium ergodic Boltzmann-Gibbs statistical mechanics does not apply. Our work considers the out of equilibrium situation of diffusion processes which yield statistical laws different from the equilibrium case.

[14] E. Barkai, R. Metzler, and J. Klafter, Phys. Rev. E 61, 132 (2000).

[15] W. Feller, An Introduction to Probability Theory and Its Applications (Wiley, Eastern New Delhi, 1969), Vol. 2.

[16] E. Barkai, Phys. Rev. E 63, 046118 (2001).

[17] E. Barkai and V. N. Fleurov, Phys. Rev. E 58, 1296 (1998).

[18] P. Allegrini, M. Bologna, P. Grigolini, and B. J. West, Phys. Rev. Lett. 99, 010603 (2007).

[19] I. M. Sokolov and J. Klafter, Phys. Rev. Lett. 97, 140602 (2006).

[20] I. Y. Wong et al., Phys. Rev. Lett. 92, 178101 (2004).

[21] N. Korabel and E. Barkai (to be published). 